\title{
Fabrizio Monaco
}

\section{Giorgio Napolitano ${ }^{1} \cdot$ Cesidio Giuliani $^{1} \cdot$ Ines Bucci ${ }^{1}$}

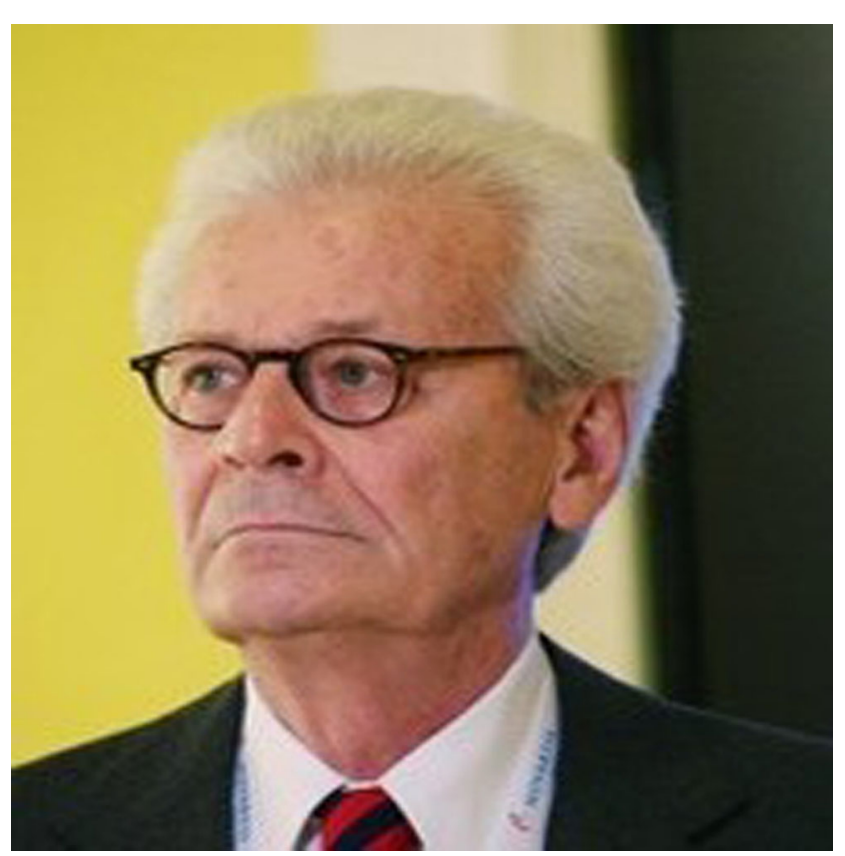

Il Professor Fabrizio Monaco si è spento improvvisamente sabato 13 luglio 2019 a Roma, dove era nato il 24 marzo 1942.

Sempre a Roma si era laureato con il massimo dei voti e la lode alla "Sapienza" nel luglio 1966, con una tesi dal titolo "Studio dell' ormonogenesi ed aspetti elettromicroscopici in alcuni casi di gozzo da difetto di ossidazione dello iodio", relatore il Professor Cataldo Cassano, tesi che nel 1967 gli

$凶$ G. Napolitano

gnapol58@gmail.com

1 Dipartimento di Medicina e Scienze dell'Invecchiamento, Università degli Studi "Gabriele d'Annunzio" Chieti-Pescara, Chieti, Italia era valsa il Premio di laurea Marzotto. Assistente Volontario presso la Patologia Medica dal 1966 al 1969, Ricercatore del C.N.R. dal 1969 al 1986, poi Professore Straordinario dal 1986 e quindi Professore Ordinario di Endocrinologia dal 1990 nell'Università “G. D’Annunzio” di ChietiPescara. Direttore della Scuola di Specializzazione in Endocrinologia e Malattie del Ricambio nel medesimo Ateneo dal 1988, anno della sua creazione. Dal 1992 è stato Direttore del Servizio Regionale per lo Screening delle Malattie Endocrine e Metaboliche della Regione Abruzzo.

Fin da studente in Medicina, il Professor Monaco ha mostrato grande interesse per ciò che avveniva nell'ambiente scientifico internazionale e la sua prima esperienza all'estero in questo ambito risale al 1964 quando frequentò la Medical Summer School in Scandinavia. Dal 1970 al 1972, conseguita una Postdoctoral International Fellowship presso i National Institutes of Health (NIH) a Bethesda, Maryland (USA), ha svolto attività di ricerca nella Clinical Endocrinology Branch del NIDDK sotto la supervisione del Dr. Jack Robbins. In quel periodo, il Professor Monaco ha stretto rapporti di collaborazione scientifica, oltre che di amicizia, con molti colleghi americani, tra i quali Ed Rall, Harold Edelhoch, Ira Goldfine, Jan Wolf, e con la comunità italiana della Branch costituita, all'epoca, da un piccolo gruppo: Nino Salvatore, Totò Aloj, Massimino D'Armiento, Bruno Bruni e numerosi colleghi che transitavano a Bethesda per periodi più brevi.

In seguito al lavoro svolto all'NIH, il Professor Monaco è stato assegnatario e Principal Investigator del Grant RO1/AM 16932 del Department of Health, Education and Welfare degli USA per gli anni 1973-1976, per lo svolgimento della ricerca Incorporation of carbohydrates into thyroglobulin in thyroid tumor e negli anni seguenti (19771980), e sempre come Principal Investigator del Grant RO/AM 16932, per la ricerca Comparative role of carbohy- 
drates into thyroglobulin, primo ricercatore della Sapienza a ottenere tale riconoscimento.

I periodi negli USA sono poi proseguiti negli anni con soggiorni prolungati, sempre all'NIH con il Dr. J. Robbins e con il Dr. Leonard D. Kohn. Per decenni la casa del Professor Monaco è stata un punto di riferimento per i colleghi americani in visita in Italia, per i loro amici e per gli amici degli amici per una "cenetta romana".

Il Professor Monaco è stato autore di numerose pubblicazioni su riviste internazionali e il suo principale interesse è stato lo studio del ruolo evolutivo e clinico della tireoglobulina. È stato inoltre Editor di molti testi a fini scientifici o didattici, sia in italiano che in inglese, incentrati sulla tiroide $\mathrm{o}$, più in generale, sull'endocrinologia. Di particolare rilevanza le cinque edizioni del trattato di Endocrinologia Clinica (Edizioni SEU) e le due edizioni del manuale Thyroid Diseases (Edizioni CRC Press).

È stato socio dell'Associazione Italiana della Tiroide (AIT), di cui è stato presidente nel 2013, della Società Italiana di Endocrinologia, della Società Italiana di Medicina Interna, della European Thyroid Association (dal 1973), dell'American Thyroid Association, dell'AME.

Queste note bibliografiche delineano il ruolo di un ricercatore e di un docente che ha dedicato la sua vita lavorativa all'endocrinologia ma non possono certo descrivere la sua figura e il rapporto che per trent'anni ci ha legato a lui.

In tutti questi anni abbiamo conosciuto il Direttore, il Maestro, ma anche l'uomo Fabrizio Monaco, tanto da riconoscerci nelle parole della figlia Olimpia nel giorno del funerale di suo padre. L'uomo Fabrizio Monaco è stato descritto con chiarezza e con una obiettività difficilmente riscontrabili in un figlio che parla di un padre e, per tale motivo, ci siamo permessi di utilizzare alcune sue frasi: "Era... un uomo energico. Categorico. Dalle incrollabili certezze. Mai quieto, in costante ricerca. Sempre impegnato a studiare, a scrivere. Un uomo che si è conquistato il suo posto nel mondo con fatica, adattandosi alle sue regole con difficoltà, come succede spesso alle persone sensibili, delicate, profonde... Ha perseguito con la tenacia di cui era capace l'obiettivo di diventare professore. Ed ha insegnato con passione e dedizione per decenni. Ha amato l'università. Che tanto gli ha dato e tanto gli ha tolto... Papà ha vissuto tutta la sua vita intensamente. Ha dato e preso. Non si è lasciato vivere. Ha sbagliato e imparato dai suoi errori. Ha amato tanto e sofferto tanto. È stato un esempio, una colonna portante della nostra famiglia, un riferimento nonostante la sua complessità e le sue grandi fragilità. Era una fonte inesauribile di conoscenze, di interessi, di studi insospettabili, di curiosità ed energie, di regali pensati, di libri suggeriti...".

Alcuni di noi hanno conosciuto il Professor Monaco negli anni ' 80 quando era ancora a Roma, al Policlinico Umberto I, altri quando si era già trasferito a Chieti. A Roma il suo regno era il laboratorio al III piano della II Clinica Medica, dove il Professore conduceva le sue ricerche sulla biosintesi e glicosilazione della tireoglobulina nei tumori tiroidei, che avrebbero portato alla pubblicazione di studi definiti "seminal" anche in articoli comparsi nei decenni successivi (es. Thibault et al., Endocrinology, 1993). A noi studenti era sconsigliato l'accesso al laboratorio perché lì "si facevano esperimenti che non avevano rilevanza nella pratica clinica". Oggi il ruolo clinico della tireoglobulina è accertato e noto a tutti. Per noi, tuttavia, la maggior parte del tempo trascorso con il Professor Monaco ha come scenario l'Università di Chieti. Quando il Professore arrivò, alla metà degli anni '80, le strutture attuali e il campus, che non hanno nulla da invidiare ad Atenei più prestigiosi, non esistevano. L'Endocrinologia che oggi ha una sede, una Scuola di Specializzazione, un Centro Regionale per lo screening delle malattie endocrine e metaboliche, una Unità Operativa Complessa, un laboratorio di ricerca presso una delle strutture più prestigiose dell'Ateneo, allora non esisteva. Le prime sedi della Cattedra di Endocrinologia sono state, in ordine di tempo, l'astanteria di un ospedale in via di dismissione e l'ex bagno per disabili di un istituto religioso. Di ambulatori o strutture sanitarie neanche a parlarne, ma il Professore ci convinse che anche in mancanza di strutture si poteva lavorare sulle persone e con il mantra "l'Università nasce senza frontiere, senza vincoli, senza legami, se non quello di fare scienza e cultura e di trasmettere la conoscenza" ci convinse che non dovevamo fermarci "all'ombra della Majella" ma che era il momento di fare un'esperienza all'estero. L'insegnamento che impartisce il Maestro non si limita alla trasmissione diretta delle sue conoscenze ma anche alla capacità di offrire la possibilità di imparare da altri. Per tutti noi il periodo all'estero è stato formativo e indimenticabile perché ci ha permesso di esplorare nuove realtà, di confrontarci con mondi diversi e di conoscere persone (Leonard Kohn in primis) che hanno cambiato il nostro modo di pensare e di essere. Il Professor Monaco ha avuto il grande merito di aver vinto le nostre resistenze e di averci quasi obbligato a migliorare e di questo non finiremo mai di ringraziarlo.

Come ha giustamente sottolineato la figlia Olimpia, il Professor Monaco era un uomo complesso e, quindi, non sempre sono state "rose e fiori": i suoi rapporti con la comunità endocrinologica hanno vissuto alti e bassi, a volte contrassegnati da un' aspra conflittualità. Il tempo ha smussato le divergenze e lenito le ferite. Allo stesso modo, anche i rari momenti di attrito vissuti nel tempo con noi si sono trasformati in preziosi insegnamenti. La stesura dei libri ha attraversato "fasi difficili"; il Professore era molto poco trattabile quando si avvicinava la scadenza della consegna dei testi perché non accettava l'idea di mancare a un impegno preso con l'editore e, nello stesso tempo, era per lui impensabile che il libro non avesse le caratteristiche che riteneva fondamentali. Ora, a distanza di anni, quei momenti che ci 
hanno formato ci mancano come e più delle parole di conforto che ci rivolgeva dopo una presentazione non brillante o un articolo rejected, perché facevano parte della sua idea di Università e del suo modo di trasmettere conoscenza.

Il Professor Monaco ha scritto numerose pubblicazioni e presentato molte relazioni ma due, in particolare, sono a noi care: nel 2007 la laudatio all'Università di Chieti per la laurea Honoris Causa a Marvin Gershengorn, e la relazione sulla tireoglobulina all' Accademia Lancisiana di Roma alla fine degli anni '80. La prima, scritta in latino ma presentata in inglese, è un excursus sul senso più profondo dell'università e di quello che dovrebbe rappresentare. La seconda, di certo non la sua presentazione più importante, ci ha insegnato che la ricerca non sempre trova un'immediata applicazione clinica ma non per questo è meno importante, come dimostrano gli studi sull'evoluzione della funzione tiroidea a partire dalla lampreda e dall'anfiosso.

Il Professore ha avuto rapporti di amicizia e stima con molti membri della comunità endocrinologica. Naturalmente il legame più forte era con i colleghi con i quali era cresciuto nella II Clinica Medica dell'Università di Roma (senza voler trascurare nessuno citiamo Enio Martino, Gianfranco Fenzi, Massimino D'Armiento), con quelli della Patologia Generale di Napoli con i quali aveva condiviso il periodo americano, dell'Endocrinologia di Pisa e con il loro direttore Professor Pinchera, che aveva conosciuto nei suoi primi passi nell'endocrinologia a Roma. Per quanto riguarda i colleghi stranieri, il Professor Monaco aveva sempre mostrato una grande ammirazione per il Prof. Jean Roche (Rettore della Sorbona), per gli americani Jack Robbins, Ed Rall, Harold Edelhoc, Len Kohn e molti altri. Anche a Chieti, nella sua facoltà, il Professor Monaco aveva ottimi rapporti con numerosi colleghi. Ad esempio, qualche tempo fa, il Professor Agostino Consoli ricordava di essere stato "accolto nell'Endocrinologia Teatina senza alcuna difficoltà o gelosia (cosa non sempre frequente) e con l'entusiasmo di chi intuiva la complementarietà delle varie sfere di azione". Il Professor Monaco è rimasto spesso in contatto con chi si era laureato e specializzato con lui a Chieti e anche con endocrinologi laureati con lui a Roma. Ad esempio, con Serafino Lio, che pur essendo uscito dall'ambiente accademico, ha continuato a collaborare con lui perché il Professore gli riconosceva "lo spirito universitario".

Per ultimo, una nostra piccola annotazione. In questo ricordo non siamo mai riusciti a chiamarlo Fabrizio, malgrado più volte ci avesse chiesto di dargli del "tu", perché per noi era e resterà sempre, con affetto e gratitudine, il nostro "Prof". 